\title{
First tritium results of the KATRIN analysis
}

\author{
Valérian Sibille* on behalf of the KATRIN collaboration \\ Massachusetts Institute of Technology \\ 77 Massachusetts Avenue Cambridge, MA 02139, USA \\ E-mail: vsibille@mit.edu
}

The KATRIN experiment aims at measuring the effective electron antineutrino mass with a $0.2 \mathrm{eV}(90 \%$ C. L.) sensitivity by analysing the $\beta$-decay spectrum of molecular tritium near its endpoint. In May and June 2018, the KATRIN collaboration took its first commissioning data using a gaseous tritium source. These data have been used to test the stability of the entire apparatus - from the windowless gaseous source to the focal plane detector - to the $0.1 \%$ level. In addition, these first commissioning runs have been successfully utilised to test the KATRIN model and ensure the reproducibility of the fitting results, which are presented in this document.

Neutrino Oscillation Workshop (NOW2018)

9 - 16 September, 2018

Rosa Marina (Ostuni, Brindisi, Italy)

${ }^{*}$ Speaker. 


\section{A direct neutrino mass measurement}

The discovery of neutrino oscillations has proved that neutrinos have mass, but the neutrino mass scale remains undetermined to this date. The neutrino mass scale may depend on the possible mass mechanisms, which may produce a degenerate $\left(m_{1} \simeq m_{2} \simeq m_{3}\right)$ or hierarchical mass spectrum, e.g. $m_{1} \ll m_{2}<m_{3}$ for the normal ordering.

From cosmological observations, stringent constraints on the sum of the neutrino masses $\sum_{i=1}^{3} m_{i}$ have been published. However, some tensions between the cosmological results themselves or local measurements exist [1]. Should the neutrino be a Majorana particle, it would experience neutrinoless double $\beta$-decay, whose decay rate would give information on the neutrino mass scale. Nevertheless, such a process remains to be observed, and even if it were, the determination of the neutrino mass scale would be hindered by the uncertainty on the nuclear matrix elements of the decay.

Direct measurements, based on kinematics constraints in $\beta$-decay (or electron capture), are model-independent insofar as the modelling of the decay spectrum is well understood. By utilising a gaseous molecular tritium source, KATRIN represents such a direct measurement, whereby the energy of the electron in the

$$
\mathrm{T}_{2} \rightarrow{ }^{3} \mathrm{HeT}^{+}+e^{-}+\bar{v}_{\mathrm{e}}
$$

decay is analysed by an eV-scale spectrometer. Paired with theoretical knowledge of the spectrum $f$ of ro-vibrational and electronic excitations of the ${ }^{3} \mathrm{HeT}^{+}$molecule [2], the kinetic energy spectrum $\frac{\mathrm{d} \Gamma}{\mathrm{d} T}$ of electrons allows the inference of the effective electron antineutrino mass $m_{\overline{v_{\mathrm{e}}}}$. The simplified differential $\beta$-spectrum $\frac{\mathrm{d} \Gamma}{\mathrm{d} T}$ for $(1.1)$, reads

$$
\frac{\mathrm{d} \Gamma}{\mathrm{d} T}(T) \propto F(T) \phi_{e}(T) \int_{0}^{Q-T-m_{\overline{\mathrm{v}}}} f(V) \phi_{V}(T+V) \mathrm{d} V
$$

with $F$ the Fermi function, $\phi_{e}$ the electron phase-space, and

$$
\phi_{v}(T)=(Q-T) \sqrt{(Q-T)^{2}-m_{\bar{v}_{\mathrm{e}}}^{2}}
$$

the neutrino phase space, which carries all the mass scale information as the kinetic energy of the electron $T$ approaches the endpoint $Q$. In (1.2), an integration is performed over all the possible excitations of energy $V$ of the ${ }^{3} \mathrm{HeT}^{+}$molecule. The current $m_{\bar{v}_{\mathrm{e}}}$ upper limit from such a direct measurement is about $2 \mathrm{eV}(90 \%$ C. L. $)$ [3, 4], and KATRIN should probe the degenerate mass scenario down to $0.2 \mathrm{eV}(90 \% \mathrm{C}$. L. $)$.

\section{A stable apparatus}

The 70m-long KATRIN setup consists of a windowless gaseous source, differential and cryogenic pumps for tritium retention, and a main spectrometer acting as a high-pass filter for the electrons collimated towards the 148-pixel silicon focal plane detector. KATRIN works in integral mode by counting the number of electrons able to overcome a given retarding potential. Each run is built by scanning several retarding potentials, thereby providing the analysts with a responseweighted integral of (1.2). On account of its scanning operation, the source activity must be stable 
within a run so as not to introduce any spectral distortions. Throughout May and June 2018, 27 three-hour runs - with the nominal source density but $1 \%$ of DT diluted in molecular deuterium, thereby corresponding to an activity approximately 200 times lower than the nominal $10^{11} \mathrm{~Bq}-$ were taken. By pressure (cf. Figure 1) and temperature (cf. Figure 2) measurements over several runs, the $0.1 \%$ source stability has been demonstrated during this commissioning campaign. Furthermore, this $0.1 \%$ stability was confirmed by the steadiness of the $21 \mathrm{kHz}$ count rate at the detector, for a retarding energy $1 \mathrm{keV}$ below the endpoint of the $\beta$-spectrum. Other investigations, e.g. the ion retention capabilities of the pumping sections, could be carried out successfully.

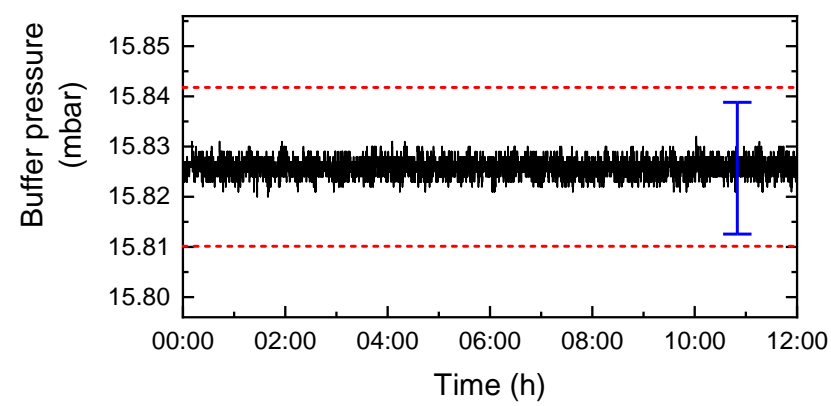

Figure 1: Pressure of the buffer feeding the windowless KATRIN source as a function of time. The red lines indicate the $0.1 \%$ stability requirements for a neutrino mass measurement, whilst the blue error represents the systematic uncertainty on the reading.

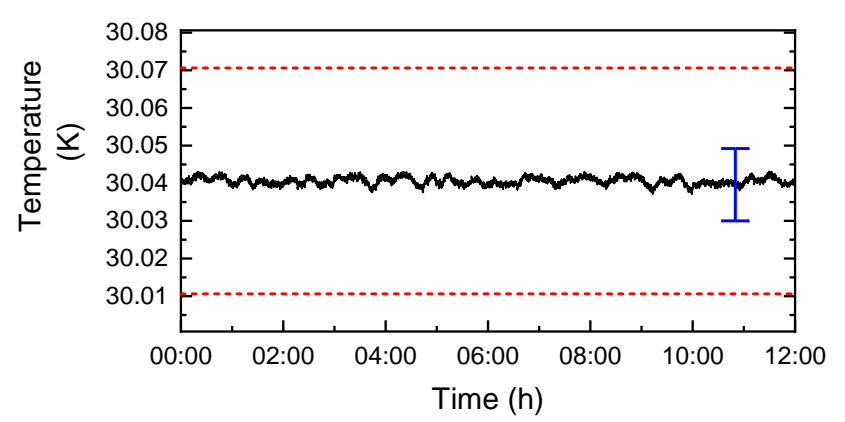

Figure 2: Source temperature as a function of time. The red lines indicate the $0.1 \%$ stability requirements for a neutrino mass measurement, whilst the blue error represents the systematic uncertainty on the reading.

\section{First tests of the KATRIN model}

For these commissioning data, the KATRIN collaboration has run both minimisers and Markov Chains to infer results about at least three parameters describing its integral measurement: the signal amplitude, the effective endpoint $E_{0}{ }^{1}$ of the $\beta$-spectrum, and the background amplitude. Because the retarding potentials are generally only seen by the $\beta$ 's from tritium decay [5], the background has been considered to be flat. For the results presented here, $m_{\bar{v}_{\mathrm{e}}}=0 \mathrm{eV}$ has been assumed. A typical fit to an integral spectrum may be found in Figure 3. As the treatment of

\footnotetext{
${ }^{1}$ The effective endpoint differs from $Q$ by the recoil of the daughter molecule and source potential.
} 
systematic uncertainties is being refined, the results from this section only include statistical uncertainties. Nonetheless, the agreement between the model and a single run over a retarding potential range of around $400 \mathrm{eV}$, which is an order of magnitude wider than originally deemed understandable, is already excellent. Moreover, the endpoint can be reproduced with a sub-eV precision for all the 27 runs, as can be seen in Figure 4.
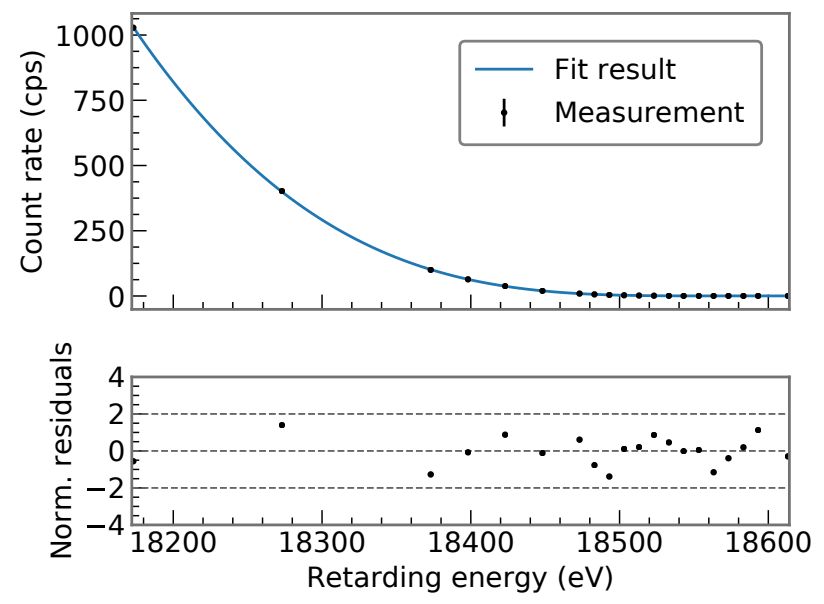

Figure 3: Best three-parameter fit of the first KATRIN three-hour tritium run with a Poisson likelihood. The residuals at best fit are also shown.

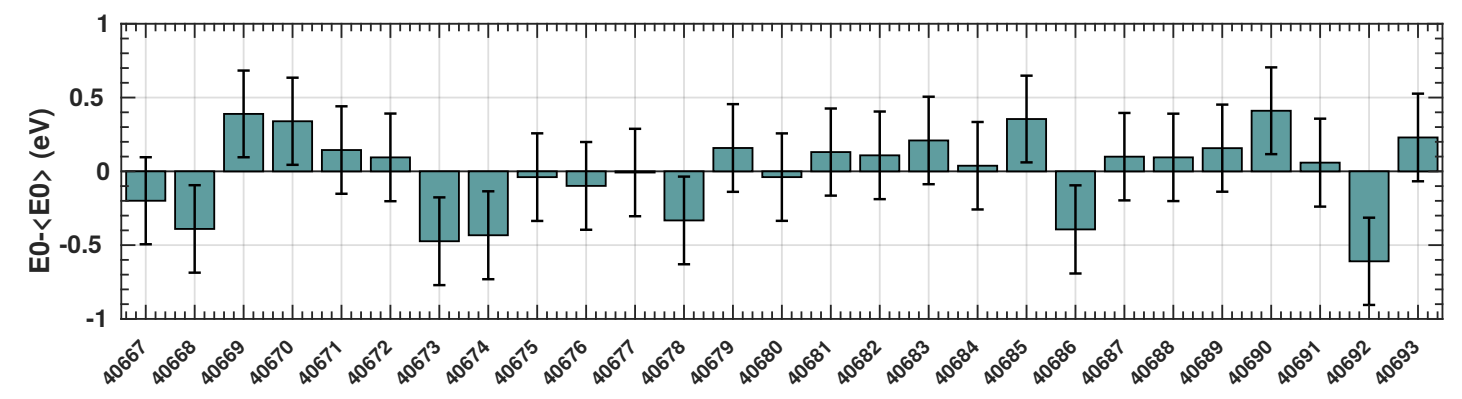

Figure 4: Relative effective endpoint $E_{0}$ of the DT $\beta$-spectrum as a function of the three-hour commissioning run fitted via a $\chi^{2}$ minimisation.

Although KATRIN is currently refining the modelling of energy losses by scattering with molecules within the source, by means of an electron gun, which will also strengthen the density model of the source, the current fit results demonstrate the robustness of the analysis chain. In addition, KATRIN has tested five blinding methods that could be used during the analysis of the forthcoming spring 2019 mass runs. Because the commissioning data are statistically limited in sensitivity to a territory already explored by the Mainz and Troitsk experiments, they provide a practical test of the feasibility of a blind analysis on actual data. It stems from these studies that spiking the data or the model with a phoney neutrino mass signal, does not affect the ability to retrieve meaningful values for the other three fit parameters. 


\section{Conclusion and prospects}

The KATRIN apparatus has been successfully tested in a closed-loop tritium operation, delivering the expected stability. Furthermore, the KATRIN model, has proved promising to explain the integrated $\beta$-spectrum measured by the experiment. Thanks to the ongoing analysis of the electron gun measurements, the study of systematics, and blinding efforts, KATRIN will be ready to present unbiased analysis results of its forthcoming neutrino mass runs next year.

\section{References}

[1] Planck collaboration, Planck 2018 results. VI. Cosmological parameters, 1807.06209.

[2] A. Saenz, S. Jonsell and P. Froelich, Improved molecular final-state distribution of het ${ }^{+}$for the $\beta$-decay process of $\mathrm{T}_{2}$, Phys. Rev. Lett. 84 (2000) 242.

[3] C. K. et al., Final results from phase ii of the mainz, neutrino mass search in tritium $\beta$ decay, The European Physical Journal C - Particles and Fields 40 (2005) 447.

[4] V. N. Aseev et al., Upper limit on the electron antineutrino mass from the troitsk experiment, Phys. Rev. D 84 (2011) 112003.

[5] KATRIN collaboration, Muon-induced background in the KATRIN main spectrometer, 1805.12173. 